and what is reported in the media drives misunderstanding. For example, the global conversation about migration is riddled with inaccurate facts and half-truths. After I finished a week-long trip to the US-Mexican border, I could envision an entire podcast series connecting what was happening on the ground with what we know about migration trends writ large. The trick is to correct misinformation but keep listeners interested enough to think about how they could fold political demography analysis into their own work or life.

My podcast is designed for maximum exposure: episodes are less than 20 minutes and produced by an expert. Because they are short, the episodes work well in a classroom setting. They also work well during a commute, and I hope that somewhere on the DC Metro there is a staffer whose attention I can hold between stops. I have consistently found that the way I walk undergraduates through new concepts and theories, give them context, and apply theories and concepts to cases that resonate is also the most effective way to reach a policy audience. That comparison is not meant to insult policy makers-or students-but rather to convey that the exercise of pitching complex topics to a sophomore-level audience is useful in multiple settings.

It also is giving me confidence to stretch into new professional areas. My podcast was born of a desire to find a novel way of teaching others about complex political issues. An unintended byproduct was that podcasting is making me a better writer. I did not expect to script my podcast but, as a listener, I am drawn to podcasts that are polished and efficient. I found that fully scripting each episode helps me to replicate my favorite shows, such as Hidden Brain on National Public Radio. Writing something that is meant to be read aloud has pushed me to be more attuned to language, pacing, and structure. I can "scratch my journalistic itch" and see my scholarly material in a fresh light, which is exactly what I need at this middle stage of my career. Just as my students have been a test audience for which jokes fall flat, which stories inspire action, and which cases provoke ire at injustice, my podcast audience tests my ability to weave such material into a coherent narrative. Although my podcast is a labor of love, I am taking what I learn about storytelling and parlaying that into new writing ventures, such as the trade book that will be published in 2021 by W.W. Norton (with the same title as my podcast). Challenging myself in these ways is keeping me motivated to push through the fatigue and talk politics even in these dark times.

\section{THE PROFESSIONAL BENEFITS OF PODCASTING POLITICS}

\section{Michael Bossetta $\mathbb{D}$, Lund University}

\section{DOI:10.1017/S1049096519001628}

Much of the hype around academic podcasting focusesrightly-on the medium's potential to make research accessible and interesting for the public. To fully realize this potential, however, more scientists need to take up the art of podcasting. But why should they?

This article spotlights the supply side of podcasting and argues that it provides three key benefits for researchers: networking, archiving, and learning. Although certainly not an exhaustive list, these valuable byproducts of podcasting are particularly beneficial for the professional development of young scholars. In outlining why, I draw on three years of experience producing the Social Media and Politics podcast. The podcast focuses on digital campaigning for elections and advocacy, and its material consists of interviews with academics and tech-industry practitioners.

\section{Networking}

Building an academic network is crucial for scholarly development. A solid academic network opens doors for knowledge exchange, publishing opportunities, and future career paths. For young scholars starting out in academia, initiating contact with more established colleagues can be a daunting task. Producing a podcast, especially an interview-based one, provides a smooth icebreaker for reaching out and conversing with scholars outside of a conference setting. Even within a conference setting, inviting a scholar to take part in a podcast provides a concrete reason to follow up and arrange an in-depth, one-onone conversation.

Moreover, the podcast medium provides networking benefits for both the interviewer and the interviewee. The interviewer (e.g., a $\mathrm{PhD}$ student) gains the opportunity to engage critically with another scholar's work while, crucially, receiving their undivided attention. Unlike an informal meeting over coffee, the interviewee (e.g., an associate professor) receives a tangible output for their time-a podcast-that can be worked into personal websites, course syllabi, and annual performance reviews.

In addition to forging new connections, podcasting affords what I call a passive form of networking. That is, by hosting a podcast in repositories such as Apple Podcasts, Spotify, or YouTube, a podcaster maintains a constant online presence across a wide range of digital channels. For young scholars in particular, this enhanced digital exposure can lead to being contacted by stakeholders within and outside of academia for various purposes, including speaking engagements and media requests that bolster a budding $\mathrm{CV}$.

\section{Social Media and Politics Podcast} SoCIALMEDIAANDPOLITICSPODCAST

\section{Y @SMANDPPODCAST}

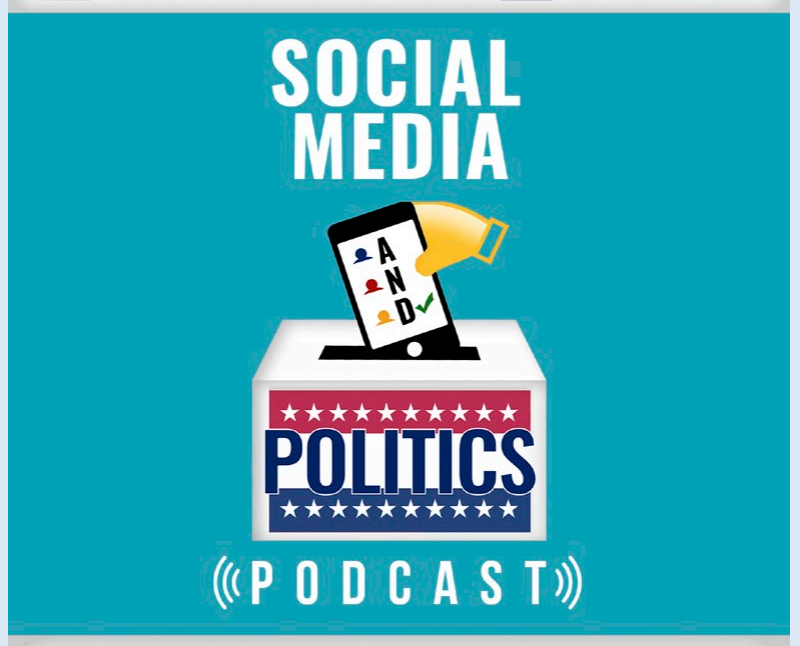

DOWNLOAD. LISTEN. LEARN. 


\section{Archiving}

Critical readers may contend that young scholars should be less concerned with networking and more concerned with producing research. Podcasting, however, is a perfect medium for gaining access to nonacademic stakeholders and archiving livestreams, Snapchat filters, Twitter bots, and promotional material such as stickers, coffee mugs, and koozies. It is fun and, through promotion, I have amassed a wide array of skills that I would not have thought to seek out otherwise (thanks, YouTube).

\section{When done thoughtfully, this co-constitutive model of knowledge production transcends personal benefits: it creates a valuable and authentic public good.}

qualitative research material. Similar to the networking benefits outlined previously, podcasting gives the researcher a reason to reach out and solicit interviews from nonacademic stakeholders; they may be more likely to participate because they receive a concrete output for their time (i.e., they may use the podcast for personal or business promotion, which indirectly benefits the podcaster). Thus, podcasting has the potential to increase a young scholar's access to industry practitioners, whose insights can be incorporated into academic research papers.

For example, I recently published a paper on social media campaigning in the 2016 US presidential election (Bossetta 2018), and podcast interviews with practitioners from the Donald Trump, Marco Rubio, and Rand Paul campaigns were key sources of qualitative data. As an additional benefit, these podcast interviews are openly accessible (a requirement for many grant funders), and they provide a resource for interested readers to delve into the full context of the conversation-not only snippets that appear in the paper.

Moreover, the open-access quality of podcasts allows research to shortcut long publishing times and circumvent journalistic paywalls. Especially for political science, disseminating research findings quickly and widely maximizes their opportunity for social impact. The aforementioned interviews, for instance, were picked up by scientists in the European Commission who were investigating the political implications of campaign microtargeting. More than benefiting my own research, these podcasts informed the work of regulators and, indirectly, contributed to the Commission's Communication, "Tackling Online Disinformation: A European Approach."

\section{Learning}

Archiving research material provides clear benefits for publishing and policy, but the primary benefit of podcasting-by far-is the vast amount of knowledge and skills that a podcaster acquires through the process. Each stage of podcast production facilitates learning and skill acquisition. Scouting new material encourages exposure to new topics while keeping abreast (or ahead) of current affairs. Preparing for an interview requires critical engagement with the work of a researcher or a practitioner. The recording process, over time, helps a podcaster hone communication skills and extract the best interview material. Even editing-the most arduous and time-consuming process-begets a valuable byproduct in that repeated exposure to an expert's insights facilitates knowledge absorption. Furthermore, publishing and promotion encourages innovation, experimentation, and creativity. I have promoted Social Media and Politics through teaching, presentation slides, Facebook
Although podcasts are a promising medium for academic outreach, the main benefactor of any podcast series is, in fact, the podcaster. As with any activity that facilitates personal embetterment, these benefits do not materialize without effort. Conservatively, I estimate that each podcast I produce takes approximately 10 hours from start to finish. Definitively, however, I can affirm that the knowledge, opportunities, skills, and satisfaction I acquire through podcasting immensely outweigh the time expended.

My intention in this article was to outline key benefits of podcasting for social scientists. I conclude, however, by stating emphatically that podcasting is not a self-serving means to an end. A podcaster, especially an academic one, should always produce content with the interests of an audience (real or intended) in mind. The art of podcasting, at its crux, is the creative process of making material that provides value to both producer and audience. When done thoughtfully, this co-constitutive model of knowledge production transcends personal benefits: it creates a valuable and authentic public good.

\section{REFERENCE \\ Bossetta, Michael. 2018. "The Digital Architectures of Social Media: Comparing Political Campaigning on Facebook, Twitter, Instagram, and Snapchat in the 2016 US Election." Journalism \& Mass Communication Quarterly 95 (2): 471-96.}

\section{A PODCASTING PRIMER}

\section{Michael Bossetta ${ }^{D}$, Lund University Leonard Williams, Manchester University}

\section{DOI:10.1017/S1049096519001707}

Although this spotlight focuses on the personal and professional aspects of podcasting by political scientists, the experiences outlined in the contributions may inspire you to create your own podcast. This podcasting primer is for those who want to follow the examples of the contributing authors or who are simply curious about the mechanics of podcasting. We first introduce key conceptual factors to consider when structuring your podcast's content and then provide guidance on the technical elements required to bring that content to life.

At the outset, all podcasts need a name, a theme, and a format. These conceptual aspects are relatively independent from one another, but they work together to create continuity in the content of a podcast series. First, the name chosen for your show "will be the number one most important identifier people will associate with you, next to your own name" (Hanks 2015, 36; 